\title{
OPTIMAL OPERATION OF DISPERSED GENERATION UNDER UNCERTAINTY USING MATHEMATICAL PROGRAMMING
}

\author{
E. Handschin * \\ Institute of Energy Systems \\ and Energy Economics \\ edmund.handschin@udo.edu \\ F. Neise ** \\ Department of Mathematics \\ neise@math.uni- \\ duisburg.de
}

\author{
H. Neumann * \\ Institute of Energy Systems \\ and Energy Economics \\ hendrik.neumann@udo.edu
}

\author{
R. Schultz ** \\ Department of Mathematics \\ schultz@math.uni- \\ duisburg.de
}

\begin{abstract}
The foreseen decentralization of the electrical energy supply has a significant impact on the overall system operation and control. To increase the economic efficiency of dispersed generation (DG) a powerful optimization technique is required. This paper presents a mathematical model including different kinds of DG units with respect to their technical characteristics as well as the optimization technique which is used to solve the problems under the existing uncertainties.
\end{abstract}

Keywords - Dispersed generation, optimal operation, mathematical programming, uncertainty

\section{INTRODUCTION}

Due to the impending renewal of the existing generation capacity on the one hand and the ongoing development of dispersed generation (DG) technology related to present decisions concerning the energy policy on the other hand DG becomes more and more important [1]. Especially the simultaneous production of thermal and electrical energy with combined heat and power units (CHP) close to the customers gains more and more importance because of the high overall efficiency. To obtain an economically optimal operation of these generation units, partially equipped with storage devices, a mathematical model and optimization is needed [2]

If several DG devices are linked together and are operated as one unit this concept is often called a Microgrid or a Virtual Power Plant (VPP) [3]. In figure 1 a schematic representation of a VPP is shown. In this example different DG units like gas turbines (GT), fuel cells (FC), other combined heat and power units (CHP), and stochastic infeed related to photovoltaics (PV) and wind turbines (WT) are connected to a VPP. As indicated in figure 1 the CHP units are connected to the electrical as well as to local heat networks. Whereas the electrical distribution networks are interconnected, the three local heat networks are isolated from each other. To transmit the optimal profiles to each DG unit and to get their actual status a bidirectional communication between the operator and each unit is needed [4].

The generation units with deterministic infeed can be controlled by the operator to supply the electricity and heat demand with minimum costs. For generation units with stochastic infeed the latter is known in terms of a probability distribution and normally the fluctuating power is fed into the distribution network. Another uncertainty consists of the electricity and heat demand of the customers. This uncertainty basically is caused by weather conditions, individual behavior and inaccuracy of the used model. These predicted demands are given in terms of $24 \mathrm{~h}$ load profiles for each customer [5].

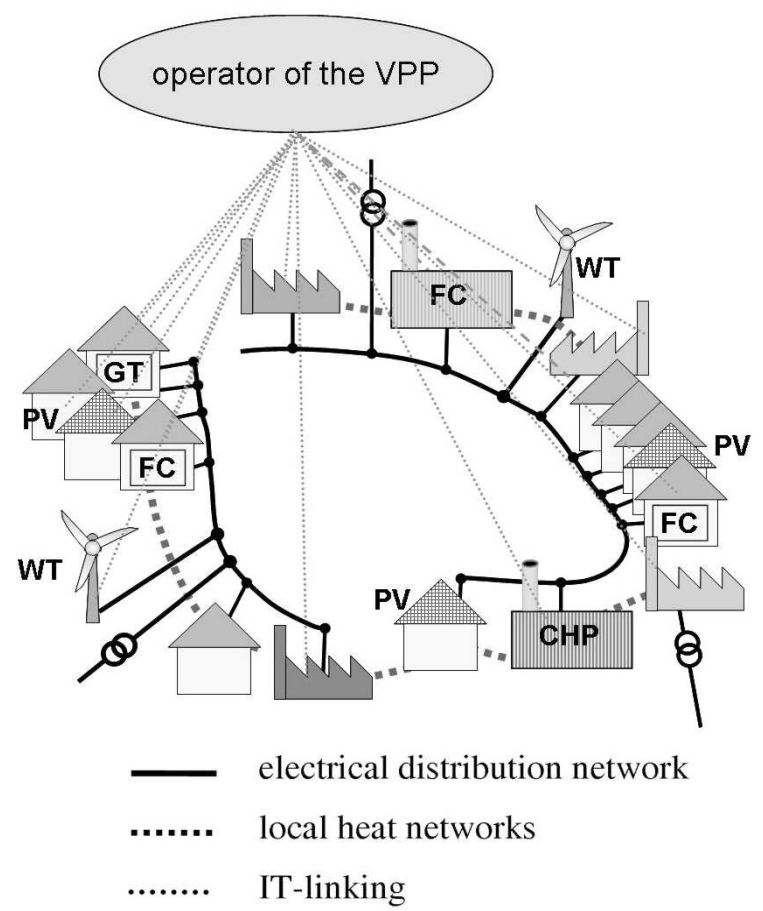

Figure 1: Exemplary representation of a VPP

\section{MATHEMATICAL PROGRAMMING TECHNIQUES}

\subsection{The Basic Model}

The basic deterministic model describes a configuration with $S$ engine-based cogeneration stations, $A_{R}$ wind turbines and $A_{W}$ hydroelectric power plants. Every engine-based cogeneration station $i$ consists of $A_{K}(i)$ boilers, $A_{M}(i)$ gas motors, $A_{T}(i)$ gas turbines as well as one thermal storage and one cooling device.

The planning horizon of $24 \mathrm{~h}$ for the management of these units is split into subintervals $t, t=0, \ldots, T$, where $m=\frac{24}{T} h$ denotes the lenght of one subinterval.

In the following description, the variables are all listed for a general power producing unit $U$. This unit can either be 
the $j$ th boiler, $K_{i j}$, in station $i$, the $j$ th gas motor, $M_{i j}$, in station $i$, the $j$ th gas turbine, $T_{i j}$, in station $i$, the $j$ th wind turbine, $R_{j}$, or the $j$ th hydroelectric power plant, $W_{j}$.

The variable $\mathbf{s}_{U}^{t} \in\{0,1\}$ indicates whether unit $U$ is in operation in time interval $t$. For cogeneration stations the variables $\mathbf{u}_{U}^{\text {up } t}, \mathbf{u}_{U}^{\text {down } t} \in\{0,1\}$ are start-up/shut-down indicators for unit $U$ at time $t$. The electricity output of unit $U$ at time $t$ is denoted by $\mathbf{p}_{U}^{t}$ and the heat output by $\mathbf{w}_{U}^{t}$.

Only for the units in cogeneration stations, there are variables $\mathbf{b}_{U}^{t}$, that describe the fuel consumption.

Furthermore, variables for the operation of the thermal storages and the cooling devices are needed: $\mathbf{a}_{i}^{t}$ and $\mathbf{e}_{i}^{t}, i=1, \ldots, S$ stand for the heat which is stored and released from the thermal storage of station $i$ at time $t$, respectively. The fill of the thermal storage $i$ at time $t$ is denoted by the variable $\mathbf{g}_{i}^{t}$, and the variable $\mathbf{k}_{i}^{t}$ is the amount of heat, which is exhausted by the cooling device of station $i$ at time $t$.

The model allows for trading within an electricity market. The variables $\mathbf{E}_{\text {reg }}{ }^{t}, \mathbf{E}_{\text {fos }}{ }^{t}$ describe the exported electricity gained from fossile or regenerative sources, and $\mathbf{I}^{t}$ is the imported electricity in time interval $t$.

The aim of the optimization is to find an operating schedule for the system with minimal costs, hence the objective function reads

$$
\begin{aligned}
\min \sum_{t=1}^{T}\left[\sum_{i=1}^{S} c \cdot\right. & \left(\sum_{j=1}^{A_{K}(i)} \mathbf{b}_{K_{i j}}^{t}+\sum_{j=1}^{A_{M}(i)} \mathbf{b}_{M_{i j}}^{t}+\sum_{j=1}^{A_{T}(i)} \mathbf{b}_{T_{i j}}^{t}\right) \\
+\sum_{i=1}^{S} c^{\text {up }} \cdot & \left(\sum_{j=1}^{A_{K}(i)} \mathbf{u}_{K_{i j} t}^{\text {up } t}+\sum_{j=1}^{A_{M}(i)} \mathbf{u}_{M_{i j}}^{\text {up } t}+\sum_{j=1}^{A_{T}(i)} \mathbf{u}_{T_{i j}}^{\text {up } t}\right) \\
& \left.+\left(c_{I} \cdot \mathbf{I}^{t}-c_{E}^{\mathrm{reg}} \cdot \mathbf{E}_{\mathrm{reg}}{ }^{t}-c_{E}^{\mathrm{fos}} \cdot \mathbf{E}_{\mathrm{fos}}{ }^{t}\right) \cdot m\right] .
\end{aligned}
$$

Here $c, c^{\text {up }}$ are the fuel costs and start-up costs for the gas-driven units. The constants $c_{E}^{\text {reg }}, c_{E}^{\text {fos }}$ and $c_{I}$ are export revenues and import costs, respectively.

The gas motors, wind turbines and hydroelectric power plants are assumed to be working at their maximum level while switched on. This gives us

$$
\begin{aligned}
\mathbf{p}_{M_{i j}}^{t} & =\mathbf{s}_{M_{i j}}^{t} \cdot p_{M_{i j}}^{\max } \\
\mathbf{w}_{M_{i j}}^{t} & =\mathbf{s}_{M_{i j}}^{t} \cdot w_{M_{i j}}^{\max } \\
\mathbf{p}_{R_{j}}^{t} & =\mathbf{s}_{R_{j}}^{t} \cdot p_{R_{j}}^{\max } \\
\mathbf{p}_{W_{j}}^{t} & =\mathbf{s}_{W_{j}}^{t} \cdot p_{W_{j}}^{\max }
\end{aligned}
$$

where $p_{M_{i j}}^{\max }, w_{M_{i j}}^{\max }, p_{R_{j}}^{\max }$ and $p_{W_{j}}^{\max }$ denote the maximum outputs.

The operation levels of the other units are limited by lower bounds $p^{\min }, w^{\min }$ and upper bounds $p^{\max }, w^{\max }$ :

$$
\begin{aligned}
& \mathbf{s}_{K_{i j}}^{t} \cdot w_{K i j}^{\min } \leq \mathbf{w}_{K_{i j}}^{t} \leq \mathbf{s}_{K_{i j}}^{t} \cdot w_{K i j}^{\max } \\
& \mathbf{s}_{T_{i j}}^{t} \cdot p_{T i j}^{\min } \leq \mathbf{p}_{T_{i j}}^{t} \leq \mathbf{s}_{T_{i j}}^{t} \cdot p_{T i j}^{\max } .
\end{aligned}
$$

Similar inequalities would apply to gas motors, wind turbines, and hydroelectric plants should they operate in intervals rather than at the above fixed levels.
The coupled production of heat and electricity in the cogeneration units has to adhere to the following proportion:

$$
\mathbf{w}_{T_{i j}}^{t}=\frac{w_{T_{i j}}^{\max }-w_{T_{i j}}^{\min }}{p_{T_{i j}}^{\max }-p_{T_{i j}}^{\min }} \cdot\left(\mathbf{p}_{T_{i j}}^{t}-p_{T_{i j}}^{\min } \cdot \mathbf{s}_{T_{i j}}^{t}\right)+w_{T_{i j}}^{\min } \cdot \mathbf{s}_{T_{i j}}^{t} \cdot
$$

Thermal and electrical load coverage is modeled via

$$
\begin{aligned}
D_{w}^{t i} & =\sum_{j=1}^{A_{K}(i)} \mathbf{w}_{K_{i j}}^{t}+\sum_{j=1}^{A_{M}(i)} \mathbf{w}_{M_{i j}}^{t}+\sum_{j=1}^{A_{T}(i)} \mathbf{w}_{T_{i j}}^{t} \\
& +\mathbf{e}_{i}^{t}-\mathbf{a}_{i}^{t}-\mathbf{k}_{i}^{t} \\
D_{p}^{t}=\sum_{i=1}^{S} & \left(\sum_{j=1}^{A_{M}(i)} \mathbf{p}_{M_{i j}}^{t}+\sum_{j=1}^{A_{T}(i)} \mathbf{p}_{T_{i j}}^{t}\right)+\sum_{j=1}^{A_{R}} \mathbf{p}_{R_{j}}^{t}+\sum_{j=1}^{A_{W}} \mathbf{p}_{W_{j}}^{t} \\
& +\mathbf{I}^{t}-\mathbf{E}_{\mathrm{reg}}{ }^{t}-\mathbf{E}_{\mathrm{fos}}{ }^{t}
\end{aligned}
$$

where $D_{w}^{t i}$ stands for the thermal load at station $i$ and $D_{p}^{t}$ for the electric load in time interval $t$.

The variables showing whether a unit $U$ is in operation in time interval $t$ have to be linked to the start-up/shut-down indicators. This is modeled by two constraints for every unit in a cogeneration station:

$$
\begin{aligned}
\mathbf{s}_{U}^{t}-\mathbf{s}_{U}^{t-1} & =\mathbf{u}_{U}^{\text {up } t}-\mathbf{u}_{U}^{\text {down } t} \\
\mathbf{u}_{U}^{\text {up } t} & +\mathbf{u}_{U}^{\text {down } t} \leq 1
\end{aligned}
$$

Furthermore, there are constraints that force these units to stay in operation until at least the given minimum up time $l_{U}$ is reached:

$$
\mathbf{s}_{U}^{t+n} \geq \mathbf{u}_{U}^{\text {up } t}, t=1, \ldots, T+1-l_{U}, n=0, \ldots, l_{U}-1 .
$$

Minimum down times can be modeled in a corresponding way, but they are not considered here.

To avoid nonlinearities in the constraints, it is assumed, that the fuel consumptions of the boilers, the gas motors and turbines are proportional to their working levels. This implies

$$
\begin{aligned}
\mathbf{b}_{K_{i j}}^{t}= & \left(\frac{b_{K_{i j}}^{\max }-b_{K_{i j}}^{\min }}{w_{K_{i j}}^{\max }-w_{K_{i j}}^{\min }} \cdot\left(\mathbf{w}_{K_{i j}}^{t}-w_{K_{i j}}^{\min } \cdot \mathbf{s}_{K_{i j}}^{t}\right)\right. \\
& \left.+b_{K_{i j}}^{\min } \cdot \mathbf{s}_{K_{i j}}^{t}\right) \cdot m \\
\mathbf{b}_{M_{i j}}^{t}= & b_{M i j}^{\max } \cdot \mathbf{s}_{M_{i j}}^{t} \cdot m \\
\mathbf{b}_{T_{i j}}^{t}= & \left(\frac{b_{T i j}^{\max }-b_{T i j}^{\min }}{w_{T_{i j}}^{\max }-w_{T_{i j}}^{\min }} \cdot\left(\mathbf{w}_{T_{i j}}^{t}-w_{T_{i j}}^{\min } \cdot \mathbf{s}_{T_{i j}}^{t}\right)\right. \\
& \left.+b_{T_{i j}}^{\min } \cdot \mathbf{s}_{T_{i j}}^{t}\right) \cdot m
\end{aligned}
$$

where $b^{\min }$ and $b^{\max }$ denote the minimum and maximum fuel consumptions. 
For the storage devices there are the following lower and upper bounds:

$$
\begin{aligned}
& 0 \leq \mathbf{a}_{i}^{t} \leq a_{i}^{\max } \\
& 0 \leq \mathbf{e}_{i}^{t} \leq e_{i}^{\max } \\
& g_{i}^{\min } \leq \mathbf{g}_{i}^{t} \leq g_{i}^{\max } .
\end{aligned}
$$

Since the losses of stored energy are proportional to the fill, the following balances interconnecting the different time intervals are used:

$$
\mathbf{g}_{i}^{t}=\mathbf{g}_{i}^{t-1} \cdot\left(1-\frac{v}{100}\right)+m \cdot\left(\mathbf{a}_{i}^{t}-\mathbf{e}_{i}^{t}\right) .
$$

Here $v$ is the percentage of lost energy.

There are also lower and upper bounds for the cooling device leading to

$$
0 \leq \mathbf{k}_{i}^{t} \leq k_{i}^{\max }
$$

Finally the following constraints for importing and exporting electricity are to be observed. While export is limited by the own production, no upper bounds are imposed on the import:

$$
\begin{gathered}
0 \leq \mathbf{I}^{t} \\
0 \leq \mathbf{E}_{\mathrm{reg}}{ }^{t} \leq \sum_{j=1}^{A_{R}} \mathbf{p}_{R_{j}}^{t}+\sum_{j=1}^{A_{W}} \mathbf{p}_{W_{j}}^{t} \\
0 \leq \mathbf{E}_{\mathrm{fos}}{ }^{t} \leq \sum_{i=1}^{S}\left(\sum_{j=1}^{A_{M}(i)} \mathbf{p}_{M_{i j}}^{t}+\sum_{j=1}^{A_{T}(i)} \mathbf{p}_{T_{i j}}^{t}\right) .
\end{gathered}
$$

\subsection{Computational Behavior of the Basic Model}

The above mixed-integer linear programming problem is readily solvable with standard software such as CPLEX [6], that uses LP-based branch-and-bound algorithms. For testing a planning horizon of 24 hours, uniformly subdivided into intervals of 15 minutes is considered. The generation system consists of 5 engine-based cogeneration stations, involving altogether 8 boilers, 9 gas motors, and one gas turbine, and of 12 wind turbines and one hydroelectrical power plant.

This leads to a mixed-integer linear program with about 14.000 binary variables, 13.000 real variables, and 22.000 constraints. Computations were carried out on a Linux-PC with a 3,0 GHz Pentium IV processor and 2,0 GB RAM.

As stopping criterion for the branch-and-bound algorithm the gap dropping below $0,01 \%$ is used. "Gap" here refers to $\frac{B S-L B}{L B} \cdot 100 \%$ where $B S$ is the objective value of the best solution found and $L B$ denotes the minimum lower LP-bound at active nodes in the search tree.

Tests with different profiles of electrical and thermal load were run. Mostly, the gap of $0,01 \%$ was reached within 30 seconds of CPU-time. Relaxing the gap to $0,1 \%$ leads to a stable reduction of CPU-time to 20 seconds and less.

\subsection{Stochastic Extension}

In the basic model complete availability of data at the moment of decision is tacitly assumed. In an operational setting, however, the latter is rarely the case since power prices, power demand, and power input from renewable resources are less and less certain over time. Typically, there is certainty about these data up to some time interval $\bar{t}$ with $0<\bar{t}<T$ followed by stochastic information in the remaining intervals. This provides the departure point for the stochastic model extension. In particular it is ensured that the decision variables belonging to the time intervals up to $\bar{t}$ are non-anticipative, i.e., do not foresee data realizations in the intervals following $\bar{t}$. The stochastic model extension will optimize these variables, reflecting here-and-now decisions under uncertainty, with respect to the expected value of the total costs they induce.

To formalize the model extension the variables in time intervals up to $\bar{t}$, also called first-stage variables, are collected into a vector $\mathbf{x}$, and the variables in subsequent intervals, referred to as second-stage variables, into $\mathbf{y}$. In view of the mentioned stochasticity, the basic model then turns into a random optimization problem fitting into the following scheme

$$
\begin{aligned}
\min _{\mathbf{x}, \mathbf{y}}\left\{c^{\top} \mathbf{x}+d(\omega)^{\top} \mathbf{y}: A(\omega) \mathbf{x}+W(\omega) \mathbf{y}=h(\omega),\right. \\
\mathbf{x} \in X, \mathbf{y} \in Y\} .
\end{aligned}
$$

As usual, dependence on $\omega$ indicates stochasticity, and the random variables are assumed to live on some probability space $(\Omega, \mathcal{A}, \mathbb{P})$. In the present situation, random power prices are captured by $d(\omega)$, random power demand by $h(\omega)$, and random power input from renewable resources by $W(\omega)$. The matrix $A$ is deterministic in the present situation. The subsequent model building and algorithmic treatment, however, handle randomness in $A$ without additional effort. The sets $X, Y$ reflect constraints in either exclusively $\mathbf{x}$ or exclusively $\mathbf{y}$. These sets are polyhedra with integer requirements to components of $\mathbf{x}, \mathbf{y}$.

With these prerequisites, the (expectation-based) twostage stochastic programming model for optimizing the non-anticipative decisions $\mathbf{x}$ reads

$$
\begin{aligned}
& \min _{\mathbf{x}}\left\{\mathbb { E } _ { \omega } \left(c^{\top} \mathbf{x}+\min _{\mathbf{y}}\left\{d(\omega)^{\top} \mathbf{y}:\right.\right.\right. \\
&W(\omega) \mathbf{y}=h(\omega)-A(\omega) \mathbf{x}, \mathbf{y} \in Y\}): \mathbf{x} \in X\} .
\end{aligned}
$$

This model aims at minimizing the expected value of the sum of the (deterministic) first-stage costs and the random second-stage costs. The latter are a function of $\mathbf{x}$ and $\omega$. They arise from an optimal selection of $\mathbf{y}$ for given decision $\mathbf{x}$ and given observation $(d, W, h, A)(\omega)$.

For algorithmic treatment of the above stochastic program the underlying random variables are assumed to be discretely distributed, with finitely many realizations (also called scenarios) $\left(d_{j}, W_{j}, h_{j}, A_{j}\right)$ and probabilities $\pi_{j}, j=1, \ldots, N$. The stochastic program then turns into the following equivalent large-scale mixed-integer 
linear program

$$
\begin{aligned}
& \min _{\mathbf{x}, \mathbf{y}_{1}, \ldots, \mathbf{y}_{N}}\left\{\sum_{j=1}^{N} \pi_{j}\left(c^{\top} \mathbf{x}+d_{j}^{\top} \mathbf{y}_{j}\right):\right. \\
& A_{j} \mathbf{x}+W_{j} \mathbf{y}_{j}=h_{j}, \mathbf{x} \in X, \mathbf{y}_{j} \in Y, \\
&j=1, \ldots, N\} .
\end{aligned}
$$

In contrast with the basic model, the above optimization problem, although again a mixed-integer linear program, is far too big to be accessible by standard solvers like CPLEX. Instead, a decomposition method is employed whose basics were laid out in [7]. The principal idea is to formulate the non-anticipativity of the $\mathrm{x}$-variables as an explicit constraint by introducing copies $\mathbf{x}_{j}, j=$ $1, \ldots, N$, and claiming $\mathbf{x}_{1}=\ldots=\mathbf{x}_{N}$. Problem (1) then turns into

$$
\begin{aligned}
& \min _{\mathbf{x}_{1}, \ldots, \mathbf{x}_{N}, \mathbf{y}_{1}, \ldots, \mathbf{y}_{N}}\left\{\sum_{j=1}^{N} \pi_{j}\left(c^{\top} \mathbf{x}_{j}+d_{j}^{\top} \mathbf{y}_{j}\right):\right. \\
& A_{j} \mathbf{x}_{j}+W_{j} \mathbf{y}_{j}=h_{j}, \mathbf{x}_{j} \in X, \mathbf{y}_{j} \in Y, j=1, \ldots, N, \\
& \left.\mathbf{x}_{1}=\ldots=\mathbf{x}_{N}\right\} .
\end{aligned}
$$

Except $\mathbf{x}_{1}=\ldots=\mathbf{x}_{N}$, all individual constraints contain variables belonging to an individual scenario $j$ only. Lagrangian relaxation of $\mathbf{x}_{1}=\ldots=\mathbf{x}_{N}$ thus leads to a decomposition into scenario-specific subproblems, which is the key to the method in [7]. This method then follows the well-known pattern of Lagrangian relaxation in integer programming: By solving a (convex) non-smooth Lagrangian dual a lower bound to the optimal value of (1) is found which is never worse the lower bound obtainable by LP relaxation of (1). Based on the results of the dual optimization, taylored heuristics derive a promising feasible solution to the (primal) problem (1). The difference between the objective value of this feasible solution and the lower bound obtained from solving the dual gives rise to a gap certificate for solution quality as already explained with the basic model. If the gap certificate is acceptable to the user the method stops, otherwise, the set $X$ is subdivided and a branch-and-bound scheme in the spirit of global optimization is put on top of the outlined Lagrangian relaxation procedure; for details see [7].

For a recent account on the state-of-the-art in stochastic programming see the handbook [8], which also has a chapter on stochastic programming applications in energy [9].

\section{ANALYSIS OF THE ECONOMIC BENEFITS OF DG}

The operation of DG is only reasonable if economic benefits compared to competing systems can be achieved. The additional investment costs of CHP plants have to be countervailed by reducing the operating costs over lifetime. The operator of those units can pursue different strategies of operating modes to obtain an economic optimum. DG can be used to supply expensive load peaks (peak shaving), which otherwise have to be balanced by cost-intensive power plants. Moreover an operating model is conceivable, which is aligned to avoid expensive regulation and reserve power by use of DG. Those units can react dynamically to variations of the expected load forecast. Furthermore they could be operated meaningfully, if the owner is able to reduce the total costs for his electrical and thermal energy demand. In this case the produced energy is used to supply the owner himself, which can lead to decreasing delivery costs for thermal and electrical energy. Operators of a VPP can be power authorities, balance responsible entities, contractors or private owner companies. The basic economic conditions finally decide, which system is the most favourable. These are the investment costs as well as the annual operating costs of all competing systems. The investment costs of CHP plants are much higher than the costs of boiler systems of the same thermal capacity. If a VPP is to be developed, additional costs for the required information technology arise. This contains an efficient energy management system to evaluate, control, and regulate the operating conditions of each individual plant as well as the communication technology for crosslinking all plants (e.g. modem, ISDN, UMTS, etc.) [10]. The annual operating costs of all systems comprise costs for purchasing electricity, heat, and fuel as well as specific maintenance costs, which are usually proportional to the produced energy. Moreover the annual interests as well as redemption of the investment and the costs for crosslinking the individual plants (e.g. connecting fees) are part of the operating costs. Incomes can be obtained by energy feeding and result directly from the remuneration of electrical and thermal energy. Assuming a thermal and electrical load forecast, the operating costs of conceivable competing systems can finally be calculated by use of current economical data.

In figures 2 and 3 the possible process of total annual costs of different systems is represented exemplarily. One system consists of conventional boilers, which cover the local heat requirement, whereby all electrical energy is imported from the distribution network. The two other systems consist of CHP plants, whereas one is operated autonomously and one as a VPP. The entire heat requirement is also covered locally and the demand of electrical energy is partly covered by own production. In figure 2 the total cumulative costs are displayed, resulting in each operational year. The clearly more favourable investment costs of the boiler systems compared to the CHP plants in year 0 can be recognized. However, the investment for a VPP is the highest one because of the additional costs for the communication technology, but the annual operating costs of CHP plants are clearly smaller than those of the boiler systems. In figure 3 the cost differences between the CHP systems and the boiler systems are presented. It is recognizable that after an operation period of 7 years the cumulative costs of the CHP plants are smaller than those of the boiler systems. 


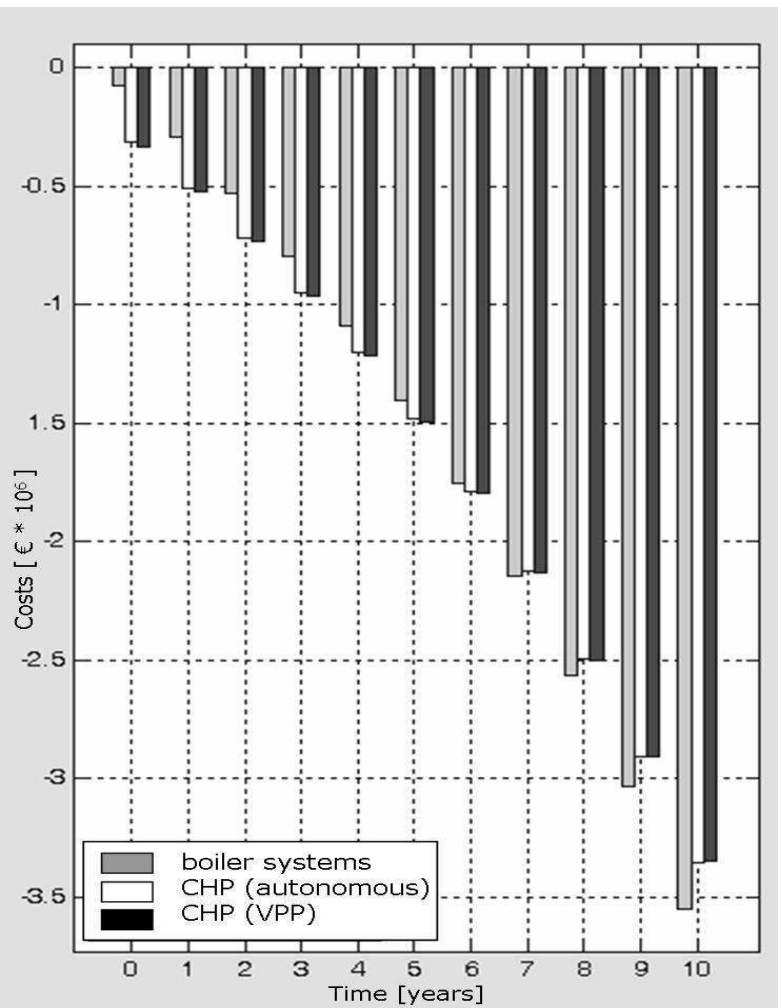

Figure 2: Total cumulative costs of three competing systems

If it is assumed, that all plants can be operated for a period of 10 years, the VPP is the most favourable alternative. At the end of the assumed lifetime it produces the lowest cumulative costs of all competing systems. That means that the additional investment costs for a VPP are profitable in this case.

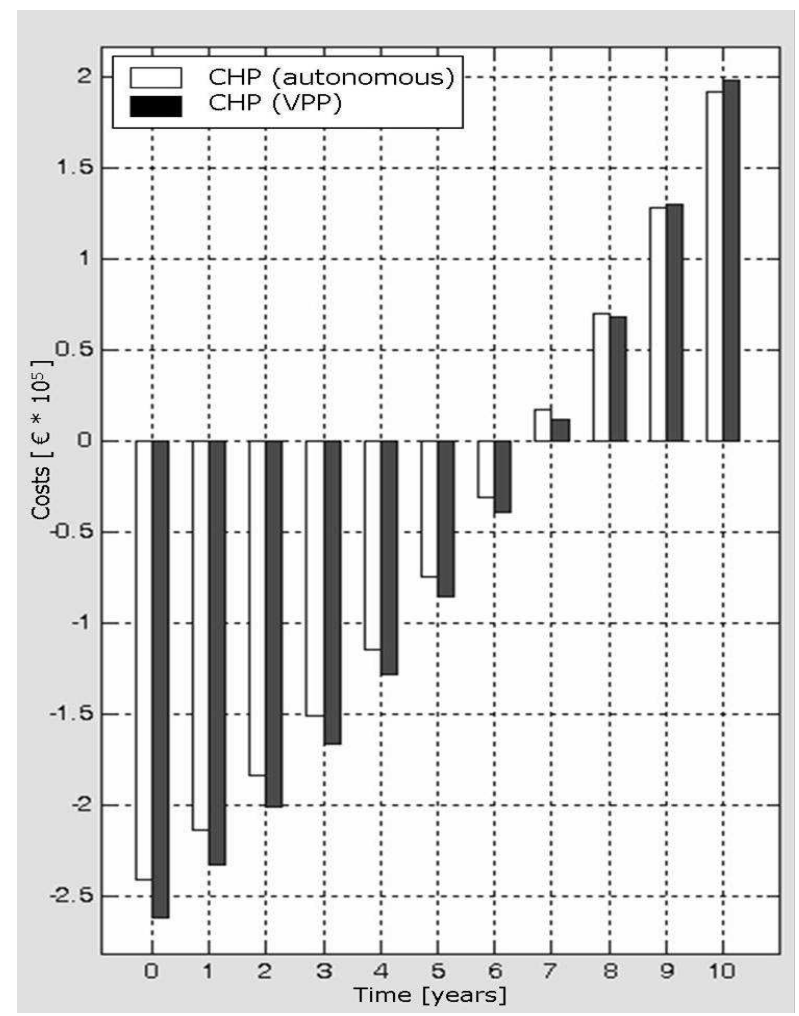

Figure 3: Cost differences between the CHP systems
If long periods are investigated, already small deviations of the relevant economic data and load forecasts can lead to strongly different results. That means that each individual case has to be analyzed under given basic conditions explicitly.

\section{RESULTS FOR A TYPICAL CONFIGURATION}

\subsection{Basic Model}

For the computational tests the VPP already mentioned in subsections 2.1 resp. 2.2 is used. The following analysis of the results is confined to one of the cogeneration stations of the VPP. This station is shown schematically in figure 4 .

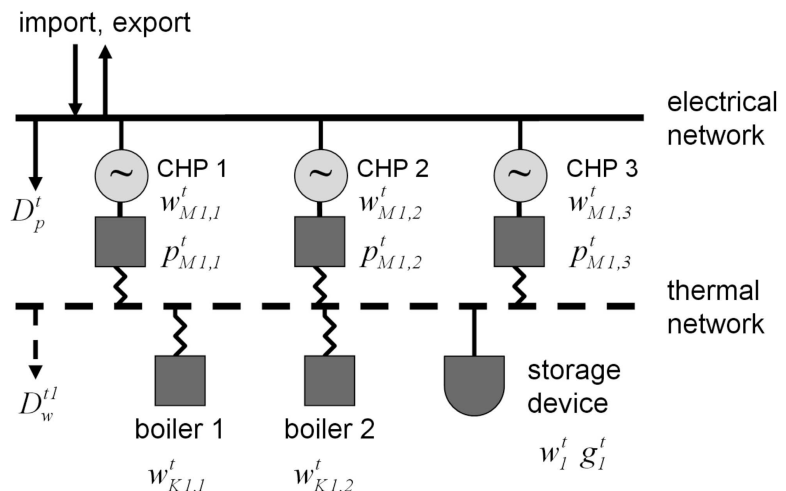

Figure 4: Cutout of a VPP, test confi guration

It consists of three CHP units, two boilers, and one thermal storage device.

Figure 5 indicates how the thermal demand at the mentioned station $\left(D_{w}^{t, 1}\right)$ is distributed to the different thermal DG units, i.e., boilers, the storage, and the CHP units, to get an over-all optimum for the entire VPP. In the figure, $\mathbf{w}_{K_{1,1}}^{t}$ and $\mathbf{w}_{K_{1,2}}^{t}$ denote the output of the boilers 1 and 2, respectively (cf. figure 4 ). Moreover, $\mathbf{w}_{1}^{t}:=\mathbf{a}_{1}^{t}-\mathbf{e}_{1}^{t}$ is the net output of the storage. In addition, figure 5 displays the total thermal output of the cogeneration station $\left(\operatorname{sum}\left(\mathbf{w}_{K}^{t}\right)\right.$ $\left.+\operatorname{sum}\left(\mathbf{w}_{M}^{t}\right)\right)$.

Figure 5 shows a substantial usage of the storage in time periods when the thermal demand drops below $6450 \mathrm{~kW}$. Then the maximum thermal output of the CHP units is insufficient to meet demand, and the added minimum output of the most cost efficient boiler exceeds demand substantially. Reducing the thermal output of the CHP implies reduction of its electrical output, such that electricity demand is left unmet, causing infeasibility or additional costs. Therefore the CHP tend to continue working at maximum or high levels, excess heat is put into the storage, and is used during time periods when the boiler is switched off, for example. Permanent storage use at high level, however, is prevented by the storage losses, that are proportional to the amount of stored energy. As one result of the optimization of the entire VPP, the most cost efficient time pattern for the boilers, storage device, and CHP units in this exemplarily considered station is found. 


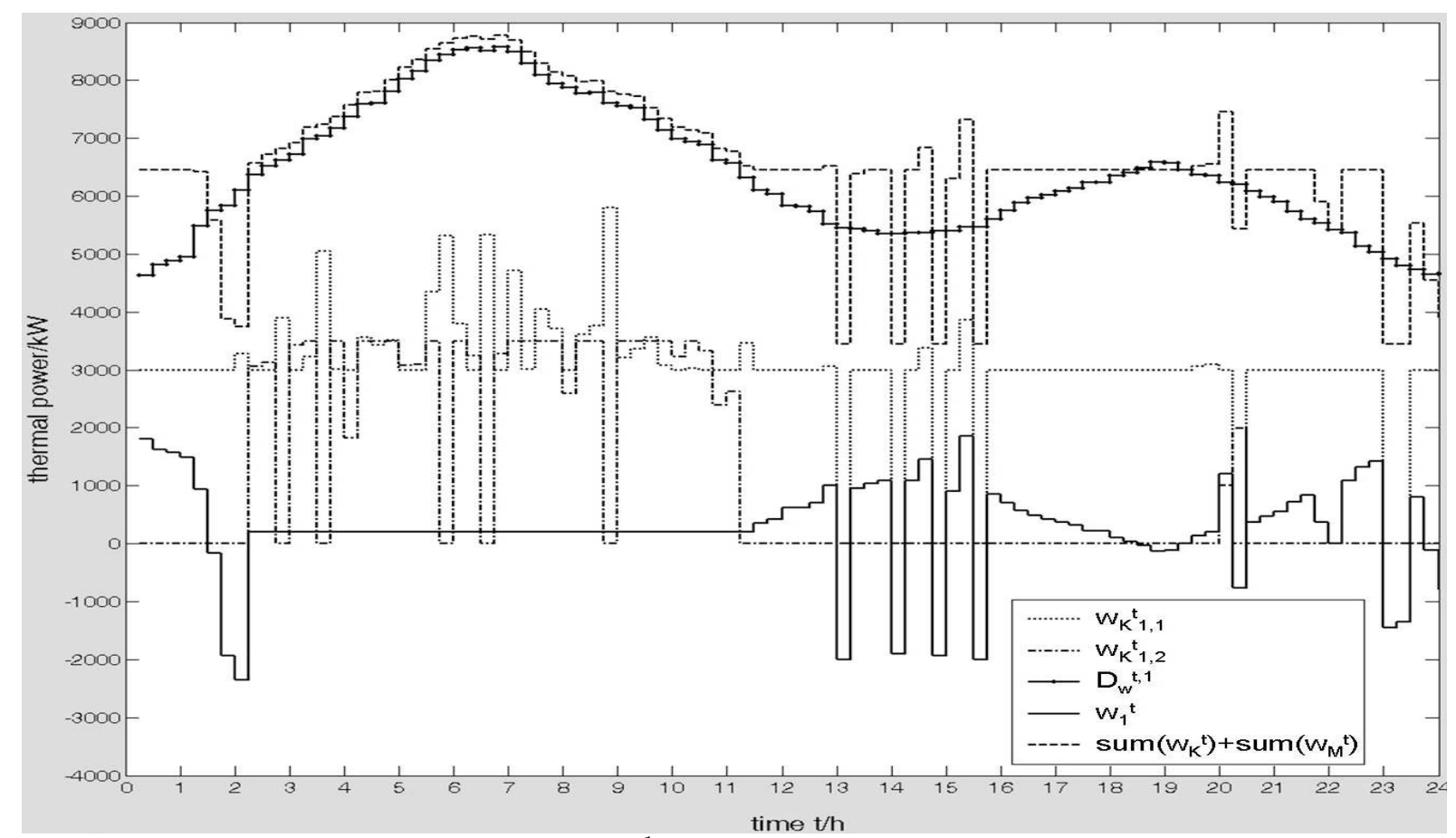

Figure 5: Optimization results of the test confi guration with $m=\frac{1}{4} h$, thermal units

\subsection{Stochastic Extension}

Initial computational tests for the stochastic extension were carried out for problem instances with random power demand. A C-implementation of the decomposition algorithm described in subsection 2.3 (dds ip [11]) was used. Again the computations were done on a Linux-PC with a 3.0 GHz Pentium IV processor and 2.0 GB RAM. Table 1 shows increased problem dimensions and computing times, for instances with 9, 11, and 31 selected scenarios:

\begin{tabular}{|l|r|r|r|}
\hline problem & \multicolumn{1}{|c|}{ A } & \multicolumn{1}{c|}{ B } & \multicolumn{1}{c|}{ C } \\
\hline \hline scenarios & 9 & 11 & 31 \\
constraints & 170179 & 207169 & 577069 \\
variables & 203412 & 247572 & 689172 \\
binaries & 106128 & 129168 & 359568 \\
\hline solution time (sec.) & 118,4 & 220,75 & 708,89 \\
gap (\%) & 0,02 & 0,02 & 0,02 \\
\hline
\end{tabular}

Table 1: Problem dimensions and computing times

This shows, that ddsip finds solutions with reasonably small gaps in acceptable solution time for these largescale stochastic mixed-integer linear programs.

The stochastic program aims at finding optimal nonanticipative first-stage decisions hedging against the various future scenarios that may occur. Table 2 shall serve as an illustration of the dispersion that different load scenarios induce on first-stage decisions, here on the thermal output of the boilers in cogeneration station 1 in time intervals 12 to 16 , if optimization is done in anticipative manner, scenario by scenario. From table 2 it becomes evident, that the optimal non-anticipative solution, i.e., the one properly reflecting the available information at the moment of decision, can not be deduced in a simple way from the collection of anticipative solutions. This optimal solution is found by the stochastic program and is displayed in the last row of table 2 .

\begin{tabular}{|c|c|c|c|c|c|}
\hline \multirow[t]{2}{*}{ scenario } & \multicolumn{5}{|c|}{$\begin{array}{l}\text { output of the boilers } \\
\text { in station } 1 \mathrm{in} \mathrm{kW} \text { in time interval }\end{array}$} \\
\hline & 12 & 13 & 14 & 15 & 16 \\
\hline 1 & 3000 & 3270 & 4731 & 4754 & 4860 \\
\hline 2 & 6500 & 3000 & 4768 & 6500 & 3000 \\
\hline 3 & 4810 & 4770 & 4731 & 4754 & 4764 \\
\hline 4 & 4810 & 4773 & 6500 & 3000 & 4860 \\
\hline 5 & 4810 & 4770 & 4731 & 6500 & 6500 \\
\hline 6 & 4810 & 4770 & 6144 & 6500 & 6500 \\
\hline 7 & 4810 & 4770 & 6500 & 6500 & 3000 \\
\hline 8 & 4810 & 4770 & 5076 & 6500 & 3000 \\
\hline 9 & 4810 & 6500 & 6500 & 3000 & 3089 \\
\hline 10 & 4810 & 6500 & 6500 & 3000 & 3089 \\
\hline 11 & 6500 & 3000 & 4768 & 6500 & 3000 \\
\hline 12 & 4810 & 4770 & 4731 & 4754 & 4764 \\
\hline 13 & 4810 & 4770 & 4731 & 4754 & 4764 \\
\hline 14 & 4810 & 4770 & 4731 & 5333 & 6500 \\
\hline 15 & 4810 & 4770 & 4731 & 6500 & 6500 \\
\hline 16 & 4810 & 4770 & 6144 & 6500 & 6500 \\
\hline 17 & 4810 & 4770 & 4731 & 6009 & 6500 \\
\hline 18 & 4810 & 4770 & 4731 & 4754 & 4764 \\
\hline 19 & 6500 & 3000 & 5837 & 6500 & 3000 \\
\hline 20 & 3000 & 3270 & 4768 & 6500 & 3000 \\
\hline 21 & 4810 & 3000 & 6500 & 3000 & 3089 \\
\hline 22 & 3000 & 3270 & 4731 & 4754 & 4860 \\
\hline 23 & 3000 & 4770 & 4731 & 4754 & 4764 \\
\hline 24 & 3000 & 3000 & 3000 & 3000 & 6500 \\
\hline 25 & 4810 & 4770 & 4902 & 6500 & 6500 \\
\hline 26 & 4810 & 4770 & 4902 & 6500 & 6500 \\
\hline 27 & 3000 & 3270 & 4768 & 6500 & 3000 \\
\hline 28 & 3000 & 4770 & 4731 & 4754 & 4764 \\
\hline 29 & 6500 & 3000 & 3000 & 4754 & 4860 \\
\hline 30 & 4810 & 4773 & 6500 & 3000 & 4860 \\
\hline 31 & 3000 & 3270 & 4731 & 4754 & 4764 \\
\hline solution & 3000 & 3270 & 4768 & 6500 & 3000 \\
\hline
\end{tabular}

Table 2: Thermal output of the boilers in cogeneration station 1 


\section{CONCLUSIONS}

The presented models and methods provide a basis for the development of a powerful tool for optimizing a coordinated operation of DG units under uncertainty of power prices, power demand, and infeed from renewable resources. By means of such a tool the operator of a VPP is able to determine its economical optimum in consideration of the relevant technical and economical constraints as well as of the different existing uncertainties.

Stochastic programming extensions to mixed-integer linear programs are the mathematical backbone of the tool. The resulting stochastic integer programs being far too big for standard solvers an advanced scenario decomposition algorithm is employed for their solution. First computational tests confirm that the decomposition algorithm is able to handle substantial problem instances with reasonable solution accuracy in acceptable time.

Altogether, the illustrated approach extends the possibilities of computer based decision support in decentralized energy markets.

\section{REFERENCES}

[1] Neumann, H.: Zukünftige Infrastruktur der verteilten Energieversorgung, Proc. of the Conference Fern-/Nahwärme und Kraft-Wärme-KopplungEnergie für Menschen mit Weitblick, Leipzig, 2004.

[2] Handschin, E.: Das virtuelle Kraftwerk für die Zukunft, Proc. of the VDI-GET Conference Entwicklungslinien der Energietechnik, Bochum, 2002.

[3] Ichikawa, T; Rehtanz, Ch.: Recent Trends in Distributed Generation - Technology, Grid Integration,
System Operation, Proc. of 14th Power Systems Computation Conference, Sevilla, 2002.

[4] Handschin, E.; Becker, R.; Uphaus, F.: Internet control for decentralized energy conversion systems, 2nd International Symposium on Distributed Generation, Stockholm, 2002.

[5] Geiger, B.; Hellwig, M.: Entwicklung von Lastprofilen für die Gaswirtschaft - Haushalte, im Auftrag des Bundesverbandes der deutschen Gas- und Wasserwirtschaft, München, 2002, www.bundesverband-gas-und-wasser.de.

[6] CPLEX Callable Library 8.1, ILOG, 2003.

[7] Carøe, C.C.; Schultz, R.: Dual decomposition in stochastic integer programming, Operations Research Letters 24 (1999), 37-45.

[8] Ruszczyński, A.; Shapiro, A. (Eds.): Handbooks in Operations Research and Management Science, 10: Stochastic Programming, Elsevier, Amsterdam, 2003.

[9] Wallace, S. W.; Fleten, S.-E.: Stochastic programming models in energy, in [8], 637-688.

[10] Arndt, U. et al.: Das Virtuelle BrennstoffzellenKraftwerk, Forschungsstelle für Energiewirtschaft München, 2002.

[11] ddsip, C package for the dual decomposition of stochastic programs with mixed-integer recourse, University of Duisburg-Essen, Department of Mathematics, 2004, www.uniduisburg.de/FB11/disma/projekte/netze/ddsip.shtml. 